\title{
ORIGINAL
}

\section{ESTRATEGIAS DE AFRONTAMIENTO ANTE EL ESTRÉS Y FUENTES DE RECOMPENSA PROFESIONAL EN MÉDICOS ESPECIALISTAS DE LA COMUNIDAD VALENCIANA. UN ESTUDIO CON ENTREVISTAS SEMIESTRUCTURADAS (*)}

\section{Vicenta Escribà-Agüir y Yolanda Bernabé-Muñoz}

Escuela Valenciana de Estudios para la Salud (EVES). Consejera de Sanitat. Generalitat Valenciana. (*) Trabajo financiado parcialmente con una ayuda del Fondo de Investigación Sanitaria (99/0704).

\section{RESUMEN}

Fundamento. Los profesionales de la medicina están particularmente expuestos a factores de riesgo psicosocial que pueden afectar su calidad de vida. La identificación de estrategias de afrontamiento frente al estrés y las fuentes de recompensa profesional permitiría instaurar medidas preventivas tendentes a moderar el efecto negativo de estos factores de riesgo. Los objetivos del presente trabajo son identificar las estrategias de afrontamiento ante el estrés que utilizan los médicos especialistas de seis hospitales públicos de la provincia de Valencia, y describir sus fuentes de recompensa y satisfacción profesional.

Métodos. Estudio cualitativo realizado por entrevistas semiestructuradas individuales a 47 médicos especialistas de seis hospitales públicos de la provincia de Valencia. Las entrevistas han sido grabadas en audio y transcritas posteriormente. Se ha realizado un análisis del contenido del discurso.

Resultados. Las estrategias de afrontamiento referidas con más frecuencia por los facultativos entrevistados, analizadas de manera general, son las centradas en las emociones y en concreto la desconexión conductual y la búsqueda de apoyo social emocional. Sin embargo, ante factores estresantes puntuales concretos de la práctica diaria, las estrategias más frecuentes son las centradas en el problema. Una parte importante de los médicos entrevistados manifiesta no tener ninguna recompensa laboral. Entre los que sí expresan elementos de recompensa, refieren en primer lugar el salario, seguido de la satisfacción personal por la dedicación a la medicina.

Conclusiones. La inclusión de formación sobre estrategias de afrontamiento activo frente al estrés en el currículo profesional de los médicos, podría aumentar sus recursos personales para hacer frente al mismo. Además se deberían instaurar cambios organizacionales que aumenten las recompensas laborales.

Palabras Clave: Personal sanitario. Estrategias de afrontamiento, recompensas laborales, estrés laboral, médicos, hospital.

Correspondencia a:

Vicenta Escribà-Agüir

EVES

C/ Juan de Garay, 21

E- 46017 VALENCIA

Correo electrónico: escriba_vic@gva.es

\section{ABSTRACT}

\section{Coping Stress Strategies and Source of Proffessional Gratification Among Specialist in the Community of Valencia. A Qualitative Study}

Background: The professionals in the medical field are particularly exposed to psychological risk factors which may affect their quality of life. Identification of stress coping strategies and the sources of professional rewards would afford the possibility of implementing preventive measures aimed a moderating the negative effect of these risk factors. The objectives of this study are to identify the stress coping strategies that are used by specialised doctors from six public hospitals of the Valencian Community, and to describe their sources of work reward and job satisfaction.

Methods: A qualitative study conducted by means of quasi-structured individual interviews of 47 specialists employed at six public hospitals in the province of Valencia. The interviews were taped and subsequently transcribed. An analysis was made of the substance of the opinions expressed.

Results. The coping strategies mentioned most often by the physicians interviewed, analyzed overall, are those focused on the emotions, specifically, disconnecting conduct and seeking social emotional support. However, when confronted with specific stressful factors in daily practice, the strategies most often employed as those focused on the problem. A major part of the physicians surveyed state not having any work reward. Among those who do state finding some aspects gratifying, top mention is made of their salary, personal satisfaction related to being in the medical field being ranked second.

Conclusions. Including training related to active stress coping strategies in the professional curriculum of physicians could heighten their personal resources for dealing with stress. Organizational changes should additionally be made to increase their work rewards.

Keywords: Health personnel. Copying Processes, Job Satisfaction. Occupational Stress. Physicians. Hospitals. Occupational Health. 


\section{INTRODUCCIÓN}

Los profesionales de la medicina constituyen un colectivo especialmente afectado por los factores de riesgo psicosocial (trabajar en continuo contacto con el sufrimiento y la muerte, la incertidumbre frente a la instauración de un tratamiento/diagnóstico, la posibilidad de cometer errores de juicio médico, etc. $)^{1}$. Ello puede afectar a la propia calidad de vida del profesional y a su entorno más próximo, y también puede tener consecuencias importantes en su desempeño laboral. Concretamente, los médicos sometidos a altos niveles de factores de riesgo psicosocial (factores estresantes laborales) tienen más probabilidades de tratar a sus pacientes de un modo más descuidado, tanto médica como psicológicamente. Así mismo, presentan una mayor propensión a realizar errores de juicio ${ }^{2}$. En un estudio reciente, la apreciación subjetiva de estrés entre los médicos encuestados fue del 91\%. Además, de éstos, el 6\% se automedicaba con sedantes y el $2 \%$ había consultado a un médico por esta causa $^{3}$.Todas estas características pueden dificultar el desarrollo de un sistema de salud de calidad.

En las últimas décadas, se ha recuperado el interés por el estudio de la influencia de las emociones y el comportamiento en el bienestar físico. Los estudios realizados ponen de manifiesto que el estrés tiene un impacto negativo sobre la salud, ya que disminuye la competencia inmunológica del individuo sometido a situaciones estresantes. No obstante, también se ha podido constatar que las estrategias de afrontamiento pueden regular dichas consecuencias negativas para la salud ${ }^{4}$.Las respuestas individuales a las situaciones estresantes dependen de una gran variedad de factores que incluyen la magnitud de la demanda, las propias características personales y recursos de afrontamiento, las restricciones personales o ambientales y el apoyo recibido por otros ${ }^{5}$. El término afrontamiento fue desarrollado fundamentalmente a partir de los años ochenta y constituye uno de los aspectos más rele- vantes desde el punto de vista psicológico en las investigaciones sobre el estrés. Las estrategias de afrontamiento se refieren a cómo se manejan las personas ante sucesos o situaciones percibidas como estresantes ${ }^{6}$. El afrontamiento no representa un concepto homogéneo; éste puede ser descrito en términos de estrategias, tácticas, respuestas, cogniciones o comportamientos. Existen diversos modelos que proponen instrumentos para su análisis 7 . Uno de los más utilizados es el de Carver y cols (1989) ${ }^{8}$. El mismo incluye dos dimensiones: a) afrontamiento centrado en el problema y b) afrontamiento centrado en las emociones. Cada una de ellas incluye diversas subdimensiones?

Otro elemento a destacar que puede actuar como moderador del impacto negativo de los factores estresantes laborales sobre el bienestar físico y psicológico de los trabajadores, son las recompensas laborales y las fuentes de satisfacción laboral. Uno de los principales modelos que analiza el papel moderador de las recompensas laborales es el modelo Esfuerzo-Recompensa de Siegrist. Éste postula que la falta de equilibrio entre «esfuerzos» y «recompensas» define un estado de distrés que puede llevar a situaciones de tensión. Un aspecto destacable es la distinción explícita que realiza entre componentes extrínsecos (situacionales) e intrínsecos (personales) del equilibrio esfuerzo-recompensa. Además, el modelo incluye características personales, como el patrón específico de afrontamiento ${ }^{9,10}$.

Dado el gran impacto de estos aspectos en el bienestar de los médicos, resulta de gran importancia determinar las estrategias de afrontamiento que éstos utilizan para hacer frente a estos factores estresantes laborales, así como las fuentes de recompensa profesional más importantes. Todo ello permitirá determinar estrategias preventivas adecuadas para controlar los factores estresantes laborales, tanto individuales como ambientales, y por tanto evitar sus efectos nocivos sobre la salud y la calidad de vida del personal médico. 
Los objetivos del presente trabajo son identificar las estrategias de afrontamiento que utilizan los médicos especialistas de seis hospitales públicos de la provincia de Valencia frente a los factores estresantes laborales, así como describir las recompensas percibidas derivadas del desarrollo de su profesión.

\section{SUJETOS Y MÉTODOS}

Diseño: En la elaboración de este trabajo se ha utilizado metodología cualitativa. La investigación se ha desarrollado a través de la técnica de entrevista individual semiestructurada. Se han establecido unos objetivos específicos previos a la realización de las entrevistas, por lo que el enfoque seguido ha sido deductivo. Se han usado categorías analíticas para describir y explicar las materias objeto de estudio.

Muestra: Con el fin de obtener una variabilidad suficiente y proporcionar mayor riqueza al análisis del discurso, se tuvieron en cuenta las siguientes variables estructurales para la elección de los participantes: el género, la edad, el estado civil, las cargas familiares, la especialidad médica, la categoría profesional y la antigüedad profesional.

Los entrevistados trabajaban en seis hospitales generales públicos de la provincia de Valencia. Un requisito para participar en el estudio fue llevar trabajando al menos durante los 15 días anteriores a la realización de la entrevista, con el fin de garantizar la vinculación con la realidad laboral inmediata de los datos recogidos durante la misma. El número inicial de informantes estimado para participar en la investigación fue de 15-20, pero se trataba de una estimación a priori, ya que la recogida de datos se iba a dar por concluida cuando saturara la información obtenida. Finalmente, el número total de médicos entrevistados fue de 47. Las especialidades de los participantes siguió esta distribución: Oncología (7), Medicina Interna (17), Traumatología (4), Radiología
(10) y Unidad de Cuidados Intensivos (UCI) (9).

Recogida de información: Tras la revisión de la literatura, el equipo investigador elaboró un guión de entrevista para recoger información sobre la características socio-profesionales, las estrategias de afrontamiento utilizadas ante el estrés, y las recompensas percibidas por los médicos en su trabajo.

Los datos sobre las estrategias de afrontamiento utilizadas fueron obtenidos de dos fuentes. En primer lugar, en un momento de la entrevista se abordó el tema de las estrategias de afrontamiento sin hacer referencia explícita al estrés. Esta constituyó la fuente indirecta, ya que, se les preguntó a los médicos por su respuesta a potenciales factores estresantes laborales citados en la literatura científica, aunque sin denominarlos de este modo. Así, se les preguntó de una manera no explícita sobre su actuación ante cuestiones concretas de la práctica profesional diaria (la incertidumbre ante un diagnóstico o tratamiento y la posibilidad de ser demandado por mala práctica). En segundo lugar, se planteó directamente el tema del estrés laboral en la profesión médica y se les preguntó a los facultativos específicamente por las estrategias que utilizaban para afrontar los efectos del estrés producido por su trabajo (fuente directa). Para obtener la información sobre las recompensas y la satisfacción laboral se plantearon dos preguntas abiertas. En primer lugar, se les preguntó de manera general sobre las fuentes de reconocimiento y gratificación obtenidas por la dedicación a su profesión. En segundo lugar, se les planteó cuáles eran las recompensas recibidas de sus superiores y de la institución en la que trabajaban por el esfuerzo y el trabajo realizados. La información de estas dos fuentes se analizó de manera conjunta, dado que el material generado para cada una de las preguntas resultó ser cualitativamente similar.

Una psicóloga especializada, miembro del equipo de investigación y coautora de 
este trabajo, realizó las entrevistas de manera presencial. Se contactó telefónicamente con los participantes con el fin de informar de los fines de la investigación y del procedimiento de la misma. Se les citó individualmente solicitándoles una disponibilidad temporal suficiente. Respecto al lugar del encuentro (el propio lugar de trabajo del entrevistado) se procuró garantizar la privacidad suficiente, así como evitar las posibles interrupciones durante el desarrollo de la entrevista. Durante el inicio de la misma se agradecía al facultativo su participación desinteresada y se solicitaba autorización para grabar la conversación. La duración de las entrevistas fue de 40 a 60 minutos y se desarrollaba según el guión planteado a priori.

Análisis: Durante la recopilación de los datos se inició el proceso de análisis. A medida que la información se iba generando, se iba llevando a cabo su estudio secuencial. Esta metodología de análisis permitió la adaptación del método de recogida a los objetivos de la investigación de una manera continua. Es decir, sirvió de evaluación continuada de la metodología utilizada. De este modo se identificaban y abordaban aspectos que permitían un tratamiento más extenso. También permitió desarrollar hipótesis a medida que se iban realizando las entrevistas, así como reformular, eliminar e incluir preguntas y temas en la estructura de entrevista planteada inicialmente. Por otro lado, fue útil para identificar la saturación de información por áreas temáticas y colectivos.

La actitud de la entrevistadora fue en todo momento participativa, facilitando su inclusión como un elemento más de la investigación. Esta visión del entrevistador como un elemento subjetivo es cada vez más frecuente en el ámbito de la investigación cualitativa, en oposición a otros enfoques que promueven una posición del entrevistador como algo externo al proceso investigador ${ }^{11}$. Esta implicación del entrevistador es inherente a la investigación cualitativa, debido a que el investigador está llevando a cabo una tarea «de campo» durante la reco- pilación de los datos ${ }^{12}$ lo cual permite una relación directa con la realidad que está estudiando. Finalizadas las transcripciones de las grabaciones en audio y notas tomadas durante las entrevistas se procedió al análisis del contenido de los datos textuales.

\section{RESULTADOS}

\section{Características de los participantes}

El número de informantes fue de 47 especialistas, de los cuales, 10 fueron mujeres. El rango de edad con mayor representación es el de 31 a 50 años, siendo el rango total de 26 a 68 años. Del total de los entrevistados, 35 están casados y 30 tienen hijos La mayor parte de los participantes tiene una experiencia laboral que va desde los 11 a los 30 años y su categoría profesional es la de adjunto.

\section{Estrategias de afrontamiento ante el estrés}

El personal facultativo hospitalario emplea una serie de estrategias de afrontamiento frente a los siguientes factores estresantes laborales: 1) la incertidumbre frente a la instauración de un diagnóstico o tratamiento, 2) la posibilidad de ser demandado por la mala práctica profesional, y 3) los efectos generales de los factores estresantes laborales.

\section{Estrategias utilizadas ante la incertidumbre frente a la instauración de un diagnóstico o tratamiento clínico}

En la profesión médica la incertidumbre constituye un sentimiento habitual. Ésta se da principalmente a la hora de establecer un diagnóstico o de instaurar un tratamiento determinado, ya que a veces resulta complicado el conocer previamente qué efectos puede llegar a tener ese tratamiento sobre un paciente concreto. Además, ningún enfermo reacciona de la misma manera y existen ca- 
sos complicados y raros ante los que la medicina no puede dar siempre una respuesta exacta. Aunque las dudas son aceptadas como característica inherente a la profesión, constituyen un elemento estresante, por lo que los facultativos se ven obligados a desarrollar estrategias para afrontarlas. Las principales estrategias utilizadas por los médicos especialistas para afrontar la incertidumbre experimentada en su trabajo hospitalario son:

Aceptación: Los médicos naturalizan un elemento estresante como es la incertidumbre hasta llegar a considerarla normal. La utilización de esta estrategia implica asumir la realidad de la existencia de esta característica como estresante.

Consultar a compañeros o a otros especialistas. Consiste en buscar ayuda, información o consejo profesional sobre qué hacer en un momento determinado.

Consultar la bibliografía. Los facultativos que pretenden ofrecer un servicio de calidad, intentan, ante la incertidumbre, que ésta sea la mínima posible. Para ello, han de contar con la mayor cantidad de conocimientos y habilidades actualizados. Para poder conseguirlo, han de estudiar y mantenerse al día en los últimos avances de su especialidad.

Actuar mediante protocolos estandarizados. Mediante esta estrategia los médicos toman decisiones y realizan actuaciones profesionales evitando al máximo la implicación personal en las mismas y procurando homogeneizar sus actuaciones.

Hablar con el paciente y su familia. En este caso, lo que se pretende es obtener simpatía, comprensión o apoyo emocional de alguien. Para los facultativos hablar con sus pacientes y/o sus familias, exponiéndoles las dudas existentes, puede suponer compartir la responsabilidad respecto a una decisión de interés para el paciente (como en el caso de la disyuntiva entre la aplicación o no de tratamientos muy agresivos), teniendo en este caso una aplicación funcional.
Estrategias utilizadas ante la posibilidad de demanda por mala práctica profesional

A la mayoría de los médicos entrevistados, les afecta poder llegar a ser demandados por algún familiar o paciente, aunque a unos pocos únicamente les sucede cuando están en urgencias, llegando algunos al extremo de estar continuamente pensando en ello. Las principales medidas que ponen en práctica los especialistas para evitar que les afecte este tema son:

Hablar con el paciente y explicarle su situación. El médico especialista expresa sus dudas y busca la comprensión de los pacientes y sus familiares. El mantener una buena relación de comunicación y confianza se considera fundamental para evitar ser demandado por mala práctica.

Practicar una medicina defensiva. En este caso, se llevan a cabo acciones activas y se ejercen esfuerzos para eliminar o paliar el factor estresante, como solicitar pruebas complementarias, aunque es una de las estrategias más extendidas también es una de las más criticadas por los propios especialistas.

"Yo hago una medicina defensiva, en muchos casos, creo que con menos exploraciones complementarias, llegaría al mismo diagnóstico». (Varón, Medicina Interna, Hospital D).

Sentir que se está haciendo un buen trabajo. Tener seguridad en el propio trabajo y saber que se está haciendo lo mejor posible constituye una estrategia para conseguir ver la situación desde una perspectiva más favorable.

«Sí que me afecta, entonces, estudio todo lo que puedo e intento hacer bien mi trabajo, el saber que puedes ser demandado no debe generarte estrés sino simplemente saber que tú estás haciendo un trabajo y ese trabajo lo estás haciendo bien, dentro de tus posibilidades». (Mujer, Radiología, Hospital D). 
Contratar una póliza de seguros. Ésta es una de las opciones elegidas por los facultativos que consideran la posibilidad de demanda algo que hay que tener en cuenta, pero prefieren evitar su influencia continua en la práctica diaria.

No tener presente la posibilidad de demanda. También existe otro grupo de profesionales a los que no les afecta o no lo tienen presente.

\section{Estrategias dirigidas a paliar los efectos generales del estrés laboral}

Cuando se les pregunta a los médicos específicamente por las estrategias que utilizan para afrontar el estrés en su trabajo, la mayoría indica:

Realizar actividades alejadas de la práctica profesional. Apartar la atención de las actividades generadoras de estrés y concentrarse en otras, alejadas de la situación laboral cotidiana, constituye el principal recurso utilizado por los facultativos para afrontar el estrés. Las actividades de ocio y las culturales permiten óptimamente este distanciamiento. El deporte, concretamente, es una de las actividades a las que se recurre con mayor frecuencia.

Pasar tiempo con la familia. Con referencia al apoyo emocional, la familia constituye el grupo de referencia que ayuda al médico a afrontar el estrés procedente de su trabajo.

Consumo de fármacos y tabaco. En momentos en que se genera mucha tensión éstas son otras de las estrategias que ayudan a reducir el estrés a los entrevistados.

Desahogarse. Otra de las tendencias expresadas por los médicos para manejar el propio malestar emocional es la acción de expresar o descargar esos sentimientos abiertamente en el momento en que se están experimentando.
Desconexión mental. El poder desconectar psicológicamente de los factores estresantes, mediante la relajación es también uno de los recursos más utilizados por los facultativos.

\section{Recompensas}

Una parte importante de los médicos entrevistados manifiesta no tener ninguna recompensa. Entre los que expresan alguna, la más recurrente es 1) el salario, seguida de 2) la satisfacción personal por la dedicación a la medicina, 3) la posibilidad que ofrece el trabajo de la puesta en práctica de las habilidades adquiridas, 4) las oportunidades de perfeccionamiento profesional, 5) la actividad docente e investigadora y 6) el reconocimiento de otras personas.

Salario. Cuando los facultativos no expresan explícitamente ninguna recompensa percibida en su práctica profesional, se hace referencia al salario recibido por el trabajo realizado como única fuente de compensación laboral.

Satisfacción personal por la dedicación a la medicina. En muchos casos, la única recompensa que obtienen es la exclusivamente personal de sentirse gratificado por el trabajo bien hecho y el poder dedicarse a la profesión que les gusta.

«Ninguna, la satisfacción únicamente es la mía personal, porque realizo un trabajo que me gusta, y el sueldo que me pagan, pero por parte del hospital ninguna». (Varón, Medicina Interna, Hospital D).

Posibilidad de puesta en práctica de las habilidades adquiridas. La totalidad del personal facultativo, a excepción de dos personas, expresan que su trabajo les permite desarrollar las habilidades en las que se han formado.

Oportunidades de perfeccionamiento profesional. La posibilidad de perfeccionar la formación de los profesionales está clara- 
mente influida por la titularidad del hospital en el que se trabaja. Concretamente, la totalidad de los médicos entrevistados que trabajan en hospitales públicos, coincide en que el hospital «no se preocupa para nada» de su perfeccionamiento profesional. Por el contrario, los especialistas que trabajan en los hospitales públicos de gestión privada se muestran satisfechos con las oportunidades que les facilita el centro en el que trabajan para su perfeccionamiento profesional.

Actividad docente e investigadora. La posibilidad de realizar tareas de investigación y docencia constituye un elemento de realización personal entre la profesión médica.

Reconocimiento profesional de otras personas. Entre las fuentes de reconocimiento profesional cabe destacar el mostrado por los compañeros y los pacientes. Los entrevistados que afirman tener algún tipo de recompensa o agradecimiento mencionan en primer lugar el sentirse apreciado y valorado por parte de sus compañeros. Otra de las fuentes de reconocimiento importantes a nivel personal está constituida por los pacientes.

«Cuando te viene a ver al cabo de 6 meses un señor, que ha estado aquí entre la vida y la muerte, y está como una rosa te da mucha satisfacción; eso es una maravilla, no pasa todos los días, pero sí que pasa bastante». (Mujer, UCI, Hospital E).

\section{DISCUSIÓN}

Este estudio se ha centrado en la información facilitada por los médicos especialistas hospitalarios respecto a la utilización de estrategias de afrontamiento ante el estrés derivado de su práctica profesional, las recompensas obtenidas y la satisfacción percibida en su trabajo. Para la obtención y análisis de esta información de carácter subjetivo, una herramienta útil es la metodología cualitativa. Esta línea de investigación, a pesar de ser relativamente infrecuente en el ámbito de la salud pública tiene una larga tradición en las ciencias sociales ${ }^{13}$.

Las aproximaciones cualitativas pueden ser un complemento de la investigación cuantitativa en el entorno sanitario ${ }^{14}$, no solamente porque permiten el estudio de áreas no accesibles a la investigación cuantitativa, sino porque además la descripción cualitativa es un requisito para una buena investigación cuantitativa, particularmente en áreas que han sido poco investigadas ${ }^{15}$. El interés entre los investigadores del ámbito sanitario en el desarrollo y la optimización de esta metodología de investigación es creciente ${ }^{11}$. De entre los métodos cualitativos, la entrevista es considerada una herramienta útil y poderosa que puede abrir muchas áreas de investigación en el ámbito médico y que ya ha sido aplicada entre la población de facultativos especialistas hospitalarios para estudiar la relación entre la vida familiar y labo$\mathrm{ral}^{16}$. Existen asimismo estudios recientes que abordan el análisis de la realidad laboral de los trabajadores de la salud mediante metodología cualitativa. Sin embargo, la mayoría de los estudios cualitativos existentes en el ambiente sanitario han sido realizados sobre el colectivo de enfermería ${ }^{17}$. Además, el volumen de trabajos realizados desde este planteamiento no es muy elevado, sobre todo en el Eestado español, por lo que nuestro estudio supone una aportación al desarrollo de esta línea de investigación.

Al analizar nuestros resultados sobre el afrontamiento teniendo en cuenta el modelo de Carver y cols. ${ }^{8}$, se evidencian dos patrones diferenciales de afrontamiento entre los médicos entrevistados.

1) Patrón de afrontamiento centrado en las emociones. Por un lado, cuando a los facultativos se les pregunta cómo afrontan el estrés experimentado en su trabajo (fuente directa), principalmente refieren estrategias centradas en las emociones. Dentro de éstas, la estrategia mencionada con más frecuencia es la desconexión conductual (realizar actividades alejadas del ambiente laboral, 
como ocio o deporte, etc.). En segundo lugar, utilizan la búsqueda de apoyo social emocional, sobretodo el pasar más tiempo con la familia o con amigos. En tercer lugar, recurren a un mayor consumo de fármacos y tabaco. En cuarto lugar, desahogarse en momentos de estrés puntual, expresando cómo se sienten. Y en quinto lugar, la desconexión mental (p.e. relajarse) constituye otro de los recursos que hacen uso los médicos para sobrellevar el estrés laboral.

2) Patrón de afrontamiento centrado en el problema. La utilización preferente de este tipo de estrategias por parte de los facultativos se da como respuesta ante cuestiones concretas de su práctica profesional (fuente indirecta). Las estrategias más utilizadas para afrontar factores estresantes laborales específicos (como la incertidumbre ante un diagnóstico), son, en primer lugar, la aceptación (p.e. aceptar la inexactitud de la ciencia médica), seguida de la búsqueda de apoyo instrumental (p. e. consultar a compañeros, consultar la bibliografía). La utilización mayoritaria de esta estrategia ha sido referida también en profesionales de la enseñanza, otro de los colectivos en los que se da una mayor incidencia de estrés laboral ${ }^{18}$.

Ante la posibilidad de demanda por mala práctica médica los médicos especialistas desarrollan también estrategias centradas en el problema que implican un afrontamiento activo (hablar con el enfermo y explicarle la situación en que se encuentra, etc.). Estos resultados coinciden con los obtenidos por otro estudio, en el que, en general, los sujetos ante situaciones estresantes, utilizan preferentemente las estrategias más adaptativas, como la planificación y el afrontamiento activo ${ }^{19}$. Así mismo utilizan estrategias centradas en las emociones, como la reinterpretación y el crecimiento (p.e. sentir que se está haciendo un buen trabajo) o la desconexión mental y la negación (p.e. no tener presente la posibilidad de demanda). El uso mayoritario de estas estrategias de entre las centradas en las emociones coincide con lo observado por otros autores ${ }^{20}$.
Valorados los resultados de manera conjunta, teniendo en cuenta la información obtenida a través de las dos fuentes (directa e indirecta), las estrategias de afrontamiento referidas con más frecuencia por los facultativos para afrontar el estrés en su trabajo son las centradas en las emociones, sobretodo la desconexión conductual y la búsqueda de apoyo social emocional. La utilización preferente de este tipo de estrategias se ha relacionado con un peor bienestar, habiéndose demostrado que aquellas personas que utilizan preferentemente este tipo de estrategias manifiestan un mayor malestar físico ${ }^{21}$.

Se ha evidenciado que el afrontamiento no solamente modera el impacto de los factores estresantes en el bienestar, sino que además influye en la percepción de las demandas ambientales como estresantes ${ }^{18}$. Específicamente, el afrontamiento activo ha resultado ser un factor protector contra el distrés psicológico ${ }^{21}$. Además, se ha demostrado que el afrontamiento centrado en el problema, actúa como un factor protector contra el distrés psicológico, mientras que el afrontamiento centrado en las emociones se ha relacionado positiva y significativamente con el distrés ${ }^{22}$.

En lo referente a las fuentes de recompensa y satisfacción profesional, la mayor parte de los médicos entrevistados manifiesta no tener ninguna recompensa. Exceptuando el salario, que constituye una compensación material, el resto de recompensas expresadas por los médicos especialistas son compensaciones no materiales. Estas recompensas, han sido descritas como elementos de satisfacción laboral entre el personal sanitario también en otros estudios ${ }^{23,24}$.

Comparando nuestros resultados con los obtenidos por Graham y cols. ${ }^{1}$, observamos, al igual que estos autores, que la satisfacción por la dedicación a la medicina puede relacionarse con la estima a la profesión y al estatus que proporciona. De manera similar, la posibilidad de la puesta en práctica de las 
habilidades adquiridas y las oportunidades de perfeccionamiento está en la línea del estímulo intelectual como fuente de satisfacción profesional referido por los mismos autores. También aparece en este trabajo como fuente de recompensa la satisfacción obtenida por el reconocimiento de otras personas, (pacientes y compañeros, principalmente).

A modo de conclusión se puede indicar que los resultados obtenidos en nuestro trabajo apoyan la idea de que para el desarrollo de la profesión médica, aunque el conocimiento adquirido en la facultad de medicina es esencial, no basta sólo con éste para el ejercicio de la misma ${ }^{25}$. La salud y el bienestar de los médicos, como seres sociales depende sustancialmente de un intercambio interpersonal favorable en términos de recompensas materiales o emocionales y de aprobación cognoscitiva ${ }^{26}$ (reconocimiento). En este sentido, y como propuesta de actuaciones futuras encaminadas a mejorar la situación laboral de los facultativos, además de cambios organizacionales que tengan en cuenta las recompensas más valoradas por ellos (como la mejora de posibilidades de promoción) y que permitieran un mayor desarrollo profesional, tal y como se ha propuesto en otros estudios ${ }^{27}$, podrían también desarrollarse propuestas formativas en materia de estrategias de afrontamiento del estrés encaminadas a incrementar el bienestar de los médicos.

\section{AGRADECIMIENTOS}

Se agradece a los participantes en el estudio, que han colaborado de forma desinteresada, aportando una valiosa información sobre su quehacer diario en el cuidado de enfermos.

\section{BIBLIOGRAFÍA}

1. Ramirez A J, Graham J Richards M A, Cull A, Gregory W M. Mental health of hospital consultants: the effects of stress and satisfaction at work. Lancet 1996; 347: 724-8.
2. Arnetz BB. Psychosocial challenges facing physicians of today. Soc Sci Med 2001; 52: 203-13).

3. Bruguera M, Gurí J, Arteman A, Valldosera JG. La atención de los médicos hacia su propia salud. Resultados de una encuesta postal. Med Clín (Barc) 2001; 117: 492-4.

4. Bayes R, Borrás FX. Psiconeuroinmunología y salud. En: Simón MA. Manual de psicología de la salud. Fundamentos, metodología y aplicaciones. Madrid: Biblioteca Nueva; 1999. p. 77-114.

5. Rabin S, Feldamn D, Kaplan Z. Stress and intervention strategies in mental health professionals. Br J Med Psychol 1999; 72: 159-69.

6. Augusto JM, Martínez R. Afrontamiento al estrés y salud. Panorama actual de la investigación. Boletín de Psicología 1998; 58: 31-48.

7. Schwarzer R, Schwarzer C. A critical survey of coping instruments. En: Zeidner M, Endler NS, editores. Handbook of coping: theory, research and applications. New York: Willey; 1996. p. 107-132.

8. Carver CS, Kumari J, Seller MF. Assessing coping strategies: a theoretically based approach. Journal of personality and Social Psychology 1989; 56: 267-83.)

9. Levi L. Stressors at the workplace: theoretical models. Occup Med 2000; 15: 69-105.

10. de Jonge J, Bosma H, Peter R, Siegrist J. Job strain, effort-reward imbalance and employee web-being: a large-scale cross-sectional study. Soc Sci Med 2000; 50: 1317-27.

11. Mays N, Pope C. Qualitative research in health care. Assessing quality in qualitative research. $\mathrm{Br}$ Med J 2000; 320: 50-2.

12. March JC, Prieto MA, Hernán M, Solas O. Técnicas cualitativas para la investigación en salud pública y gestión de servicios de salud: algo más que otro tipo de técnicas. Gac Sanit 1999; 13. 312-29.

13. Meyer J. Using qualitative methods in health related action research. Br Med J 2000; 320: 178-81.

14. Mays, N, Pope C. Qualitative Research: Rigour and qualitative research. Br Med J 1995; 311 : 109-12.

15. de Andrés J. El análisis de estudios cualitativo. Aten Primaria 2000; 25: 42-6. 
16. Dumelow C, Littlejohns P, Griffiths S. Relation between a career and family life for English hospital consultants: qualitative, semistructured interview study. Br Med J 2000; 320: 1437-40.

17. Más R, Escribà V, Cárdenas M. Factores estresantes laborales percibidos por el personal de enfermería hospitalario: un estudio cualitativo. Arch Prev Riesgos Labor 1999; 2: 159-67.

18. Griffith J, Steptoe A, Cropley M. An investigation of coping strategies associated with job stress in teachers. Br J Educ Psychol 1999; 69: 517-31.

19. Crespo M, Cruzado JA. La evaluación del afrontamiento: adaptación española del cuestionario COPE con una muestra de estudiantes universitarios. Análisis y Modificación de Conducta 1997; 23: 797-830.

20. Brissette I, Scheier MF, Carver CS. The role of optimism in social network development, coping, and psychological adjustment during a life transition. J Pers Soc Psychol 2002; 82: 102-11.

21. Margallo-Lana M, Reichelt K, Hayes P, Lee 1, Fossey J, O'Brien J, Ballard C. Longitudinal comparison of depresion, coping, and turnover among
HHS and private sector staff caring for people with dementia. Br Med J 2001; 32: 759-70.

22. Higgins J, Endler NS. Coping, life stress, and psychological and somatic distress. European Journal of Personality 1995; 9: 271-281.

23. Fernández MI, Villagrasa JR, Gamo MF, Vázquez J, Cruz E, Aguirre V, Andradas V. Estudio de la satisfacción laboral y sus determinantes en los trabajadores sanitarios de un área de Madrid. Rev Esp Salud Pública 1995; 69: 487-97.

24. Fernández MI, Moinelo A, Villanueva A, Andrade C, Rivera M, Gómez JM, Parrilla O. Satisfacción laboral de los profesionales de atención primaria del área 10 del Insalud de Madrid. Rev Esp Salud Pública 2000; 74: 139-47.

25. Pedraza V. Formación médica y necesidades sociales. Med Clín (Barc) 2001; 116: 658-63.

26. Siegrist J, Fernández JA, Hernández R. Perspectiva sociológica de la calidad de vida. Med Clin (Barc) 2000; 114: 22-4.

27. Simó J, García MA. Elementos relacionados con la efectividad y eficiencia del trabajo del médico de familia: un estudio Delphi en la provincia de Alicante. Gac Sanit 1999; 13: 282-91. 